\title{
„DIE FENSTER MEINER POESIE SIND WEIT GEÖFFNET ZUR STRASSE“. EIN GESPRÄCH MIT ROGER PERRET ÜBER SEINE ANTHOLOGIE MODERNE POESIE IN DER SCHWEIZ
}

Roger Perret gab 2013 eine epochale Anthologie mit dem Titel Moderne Poesie in der Schweiz heraus, die auf großes Interesse stieß und auch schon mehrfach ausgezeichnet wurde. 600 Gedichte von 255 AutorInnen und zwar nicht nur in vier Landessprachen der Schweiz (Deutsch, Französisch, Italienisch und Rätoromanisch), sondern auch in anderen Sprachen, in denen die Literatur in der kulturell sehr bunten Schweiz entsteht, dokumentieren eine enorme Vielfalt der Poesie in der Schweiz zwischen 1900-2013. Der Ausdruck Poesie ist für Perret sehr weit gefasst und daher finden wir in der Anthologie nicht nur Gedichte im traditionellen Sinn. Perret, der Philosophie, Literaturkritik und Komparatistik studierte, arbeitete als Projektleiter in der Abteilung Darstellende Künste und Literatur bei der Direktion Kultur und Soziales des Migros-Genossenschafts-Bundes in Zürich. Er ist Herausgeber der Werke von Nicolas Bouvier, Alexander Xaver Gwerder, Hans Morgenthaler, Annemarie Schwarzenbach, Sonja Sekula und Annemarie von Matt und Mitherausgeber des Hörbuchs Wenn ich Schweiz sage... Schweizer Lyrik im Originalton von 1937 bis heute (2010).

Roger Perret stellte seine Anthologie Moderne Poesie in der Schweiz im Sommer 2014 auf dem Festival Pranger in Rogaška Slatina vor und damals entstand folgendes Gespräch, in dem er die Hintergründe der Entstehung seines monumentalen Werkes erklärt, einiges zu seiner Auswahl sagt und auch hinter die Kulissen blicken lässt.

„Es gibt keine Einheit mehr", heißt es bereits im ersten Gedicht mit dem Titel „Kontraste“ von Blaise Cendrars und auch in Ihrem Nachwort mit ebenso vielen „Sätzen“, wie es Kapitel im Buch gibt - 28. Das Gedicht "Kontraste" sehen Sie als Beginn der modernen Poesie in der Schweiz an und Blaise Cendrars steht neben Robert Walser und Adolf Wölfli. Was verbindet diese drei Klassiker der Moderne?

Dass es keine Einheit mehr gibt, heißt zum Beispiel, dass man sich nicht mehr an formale Vorgaben gebunden fühlt. Man schreibt also nicht mehr ein Gedicht in einer gebundenen Form, ein Sonett oder in einem Versmaß. Die Form ist keine Verpflichtung mehr. Man kommt ins Offene, wie Hölderlin einmal geschrieben hat: „Komm! ins Offene, Freund!“ Das andere ist, dass der Beginn der lyrischen Moderne, den ich auf ungefähr 
1900 veranschlagt habe, im Kontext der künstlerischen Moderne (Malerei, Musik, Architektur usw.) anfangs des 20. Jahrhunderts anzusiedeln ist. In der Malerei kommt zum Beispiel der Kubismus - da gibt's keine Einheit mehr, jedoch eine Multiperspektive - das ist ein Begriff, der auch in Cendrars' Gedichten zu beobachten ist. Es gibt nicht mehr ein lyrisches Ich, das dirigiert, sondern man collagiert, montiert fremde Verse oder Reklamesprüche in ein Gedicht hinein, lässt Satzzeichen weg usw. In einem Gedicht ist alles möglich. Es ist nicht mehr eine verbürgte Sprache, die nur vom Autor stammt, sondern man nimmt von da oder da. Bei Cendrars gibt es ein Gedicht „Menü“ mit Anweisungen zum Essen und, wenn ich richtig informiert bin, sind das Zitate aus einem fremden Buch. Die hat er einfach raus genommen und zusammengeführt zu einem Gedicht.

Die Texte dieser drei Autoren brechen Regeln, grammatische, aber auch solche der Perspektive oder des Geschmacks. Das ist etwas Neues. Neu war auch, dass Walser und Wölfli die Mundart produktiv eingesetzt haben. Die Qualität ihrer Texte hängt auch von der Vermischung der Mundart mit dem Hochdeutschen ab. Einige Arbeiten verwenden die beiden Idiome sogar abwechslungsweise. Und Wölfli kann als der erste moderne Mundartdichter der Schweiz bezeichnet werden. Er war ja ein ungebildeter Knecht, der erst als Patient in einer psychiatrischen Klinik zu schreiben begann. Doch welch tiefsinnige Texte hat er geschrieben, mit zum Teil neuartigen formalen Mitteln. Wichtig ist auch, dass er und Walser alltägliche, ja banale Themen zur Sprache bringen und sich von einer reinen dichterischen Sprache, wie sie im 19. Jahrhundert verwendet wurde, verabschiedet haben.

Ihr Buch heißt Moderne Poesie in der Schweiz. Dabei hat Max Frisch einmal so etwas gesagt: Wir Schweizer sind langsam. Etwas Neues beginnen wir erst, wenn die anderen es schon ausprobiert haben. In Ihrer Anthologie sieht man jedoch, dass die Schweizer parallel zu den Expressionisten Neues geleistet haben. Das Gedicht „Kontraste“ von Cendrars entstand 1913 und das Gedicht „Weltende“ von Jakob van Hoddis, das wir als Beginn des Expressionismus ansehen, 1911. Also waren die Schweizer überhaupt nicht so spät dran?

Nein. Obwohl: Cendrars war ein Schweizer, hat aber in Paris gelebt und gearbeitet, wird von den Franzosen auch als eigener Autor wahrgenommen.

\section{Wie viele andere auch?}

Genau. Aber das ist eine der Eigentümlichkeiten auf der Suche nach der Moderne in der Schweiz. Wenn man die früheren Anthologien mit Lyrik aus der Deutschschweiz anschaut, dann findet man als moderne Autoren Walser und vielleicht noch Albin Zollinger. Viele Gedichte sind jedoch von zweitklassigen Autoren, die wie Epigonen zum Beispiel des Expressionismus wirken. Eine Ausnahme ist Hans Morgenthaler, den ich sehr schätze, weil er ganz direkt, in einer Art unkünstlerischen Sprache geschrieben hat, die heute noch frisch wirkt. Einige Lyriker waren Nachahmer von Rilke, den man ja auch 
zur Moderne zählt, obwohl seine Sprache heute für uns, wenn man Rilke z. B. mit Brecht vergleicht, manchmal etwas gestelzt wirkt. Aber Rilke hätte sich auch nicht als Avantgardist bezeichnet. Viele Schweizer Autoren damals kopierten also den Rilke-Ton oder den expressionistischen Ton, schrieben aber ihre epigonalen Gedichte erst viel später, in den 20er und 30er Jahren, ja sogar noch später. Der Expressionismus als innovative literarische Strömung fand jedoch vor dem Ersten Weltkrieg statt!

Auf der Suche nach modernen Dichtern bin ich auf Persönlichkeiten gestoßen, die Patienten in psychiatrischen Kliniken waren und sich dort künstlerisch betätigt haben. Die haben modernere Texte geschrieben als die meisten Autoren vor dem Zweiten Weltkrieg, die sich als Lyriker verstanden haben. Vielleicht gerade weil sie, wie auch die Doppelbegabungen (Maler-Dichter wie Paul Klee oder Hans Arp), nicht so feste Begriffe von einem Gedicht gehabt haben. Sie gingen viel spontaner und unbelasteter mit dem Sprachmaterial um.

\section{Viel offener?}

Ja. Und sie haben Arbeiten geschaffen, die besser überdauert haben und eigentlich moderner waren, weil sie nicht versucht haben, irgendeinen Ton zu kopieren.

Wie erklärt es sich, dass die Dadaisten ausgerechnet im braven Zürich auftraten: Hans Arp und Hugo Ball z. B.?

Ja, wie kann man das erklären? Weil Zürich damals (und heute) ein weltoffener Ort war und weil die Leute deswegen dorthin geflüchtet sind. Natürlich: Zürich war die größte Stadt in der Schweiz, und man ist in die Schweiz gegangen, weil unser Land neutral war. Und so sind einfach verschiedene Autoren aus verschiedenen Ländern, Tristan Tzara aus Rumänien, Hans Arp aus dem Elsass oder Hugo Ball und Emmy Hennings aus Deutschland zusammengekommen und haben, als Reaktion auf den Wahnsinn des Kriegs, einen Umbruch der Werte versucht und die Sprache beim Un-Sinn-Wort genommen. Um das zu realisieren, brauchte es vermutlich gerade diese Spannung zwischen dem Krieg außerhalb und dem Frieden innerhalb der Schweiz. Es wäre vermutlich schwierig gewesen, diese künstlerische Revolution in der Atmosphäre, wo Krieg herrschte, zu machen.

\section{Wo man um etwas anderes kämpfen musste.}

Ja. Wenn sie vielleicht in Paris oder Berlin gewesen wären, mit den Kriegsängsten, da hätte man vielleicht andere Schwerpunkte gesetzt. Aber ich bin mir da nicht so sicher.

In Ihrer wunderbar zusammengesetzten Anthologie gibt es sehr viele fast unbekannte Autoren. Es gibt z. B. eine Dichterin Constance Schwartzlin-Berberat, mit der Ihrer Meinung nach zeitlich die moderne Poesie beginnt.

Ja. Sie ist eine Pionierin der Moderne. 
Sie vergleichen sie mit Guillaume Apollinaire und mit Stéphane Mallarmé und heben den kalligraphisch-bildnerischen Aspekt ihrer Arbeiten hervor. Können Sie uns in Umrissen vorstellen, warum sie so wichtig ist?

Ich glaube, gewisse Leute waren da schon überrascht, denn man hat, außer in der Art BrutForschung, noch nie etwas über sie gehört. Ich bin aber nicht der erste, der sie entdeckt hat. In den 90er Jahren ist ein Buch einer Westschweizer Medizinhistorikerin erschienen. Sie hat das bis jetzt einzige Werk über Constance Schwartzlin-Berberat veröffentlicht. Ich habe als Literaturspezialist in der Migros-Kulturförderung gearbeitet, und wir haben dieses Buch unterstützt. So bin ich auf sie gestoßen. Sie hat mich damals schon beeindruckt, aber erst als ich Originale im Archiv in der Waldau, in der psychiatrischen Klinik, gesehen habe, habe ich gemerkt, was wirklich dahinter steckt: Denn das Eigentümliche an ihrem Werk ist, dass es in einer kalligraphischen Handschrift geschrieben ist, in so genannten „Cahiers“. Darin sind die Texte angeordnet zum Teil wie visuelle Gedichte. Man findet darin verschiedene Schriftgrößen, verschieden große Abstände zwischen den Wörtern und Zeilen, aber es gibt auch graphische Zeichen, die habe ich nicht übernehmen können. Man müsste eigentlich ein Faksimile machen, um wirklich zu zeigen, wie sie gearbeitet hat, wie ein Text in seiner visuellen Gestalt aussieht. Zum Beispiel kann auf einer Seite nur ein Wort stehen, in Großbuchstaben geschrieben: „NON“. Nur das. Aufgrund der Vorlage versuchte ich, diese besondere graphische Gestaltung zu übernehmen. Das hat die erwähnte Medizinhistorikerin nicht gemacht. Sie hat die Texte einfach nur als Wortlaut transkribiert, ohne die komplexe typographische Struktur des Manuskriptes zu reproduzieren. Dadurch geht meines Erachtens gerade das Entscheidende dieser Texte verloren. Die Texte von Constance Schwartzlin-Berberat sind aber auch deshalb ungewöhnlich und modern, weil sie eine ,fortgesetzte Reflexion über die Sprache“ sind. Und weil sie nach der Beziehung zwischen den verrückt genannten Patienten und den sich als normal verstehenden Ärzten fragen, in einer Sprache, die buchstäblich ver-rückt ist. Schwartzlin-Berberat hat auch über die Nahrung zum Beispiel geschrieben und ein Kochbuch hinterlassen. Sie hat aber manchmal nichts gegessen, musste deshalb zwangsernährt werden, was im Hinblick auf die von ihr formulierten Sprachmenüs fast ein Hohn ist.

\section{Paradox?}

Ja, paradox. Ich habe das Wortküche genannt. Sie hat mit Worten gekocht, aber nicht unbedingt für den körperlichen, sondern für den geistigen Leib. Und wie gesagt, diese Texte erinnern formal ein wenig an die Calligrammes von Apollinaire oder an das berühmte Buch Un coup de dés ... von Mallarmé, wo auch das Graphische, das Visuelle eine große Rolle spielt. Man liest einen Text dann anders. Und bei ihr ist es auch so. Man liest dann den Text nicht wie üblich von oben nach unten und von links nach rechts, sondern man kann ihn manchmal auch von rechts nach links lesen oder nur vertikal. Also die Wörter nur untereinander gelesen, ergibt einen anderen Sinn. Das ist wie in einem Text von ihr, 
wo die Anschauung des Himmels und des Firmaments mit der Erschaffung der Buchstaben und Wörter in Verbindung gebracht und diese Beziehung als eine Art Paradies bezeichnet wird. Als würden die Wörter wie Sterne am Himmel Konstellationen bilden, die man mit unterschiedlichen Lesarten immer wieder neu erschaffen kann. Da haben wir wieder die Multiperspektive. Es gibt nicht nur einen Zugang, sondern verschiedene.

\section{Eine ganz interessante Dichterin.}

Ja. Eine sehr interessante, die, das muss man auch sagen, natürlich nicht wusste, dass sie modern war. Das ist ja gerade das Besondere. Sie hat, das weiß man, keine moderne Literatur gelesen. Sie hat vermutlich überhaupt nicht gelesen. Sie war eine Arztgattin, die verrückt geworden ist, weil ihr Mann und ihre Mutter im gleichen Jahr gestorben sind. Dann ist ein schizophrener Schub ausgebrochen. Bei Wölfli weiß man mehr. Er hat Zeitschriften und vielleicht auch Bücher gelesen, aber ich glaube nicht, dass Wölfli zum Beispiel Robert Walser gelesen hat. Er hat die moderne Literatur nicht gekannt. Schwartzlin-Berberats und Wölflis Kunst war eher Ausdruck ihrer seelischen Befindlichkeit oder ihrer existenziellen Krise. Trotzdem und obwohl sie ihre Arbeit nicht als modern verstanden, haben sie ähnliche künstlerische Mittel verwendet wie die modernen Künstler und Dichter.

\section{Und Alfonsina Storni?}

Das ist ein anderer Fall. Alfonsina Storni ist als Kleinkind mit ihren Eltern nach Argentinien ausgewandert und hat dann zur Schweiz keine große Beziehung mehr gehabt. Sie hat sich in Argentinien als Lehrerin durchgeschlagen und ist dann eine ganz bekannte südamerikanische Dichterin geworden, eine der wichtigen neuen Stimmen in den 20er, 30er Jahren. Einmal hat sie aber die Schweiz besucht. Es gibt ein Gedicht darüber, wo sie auf ihren Geburtsort im Tessin anspielt.

\section{Vor kurzem ist ein Buch über sie erschienen.}

Ja. Von Hildegard Elisabeth Keller. Im Tessin schaut man sie schon ein wenig als eine eigene Dichterin an, obwohl sie auf Spanisch geschrieben hat.

\section{Aber was hat dazu geführt, dass Sie sie auch in Ihre Anthologie aufgenommen haben?} Das war ein Grundsatzentscheid, es heißt ja nicht „Moderne Schweizer Poesie“, sondern „Moderne Poesie in der Schweiz“.

\section{Aber sie lebte in Argentinien.}

Sie war in Argentinien, aber sie hat einen Bezug zur Schweiz, ist hier geboren. Das Nationale stand nicht im Vordergrund. Ich wollte auch DichterInnen vorstellen, die aus dem Ausland stammen und trotzdem die poetische Landschaft unseres Landes geprägt haben. 
Aber natürlich, Alfonsina Storni ist eine Ausnahme. Ich würde sie nicht als Schweizer Dichterin, aber als Dichterin mit Schweizer Herkunft bezeichnen.

In diesem Zusammenhang, den Sie jetzt erwähnt haben, steht, dass Sie auch sehr viele Einwanderer aufgenommen haben - nicht nur die Schweizer. Sie sagten soeben, dass das Nationale nicht die wichtigste Rolle spielt. In der Schweiz gibt es ja über $20 \%$ Einwanderer. Welche Rolle spielen sie in der modernen Poesie in der Schweiz?

Zu den Einwanderern gehören Autorinnen wie Dragica Rajčić, die aus Kroatien stammt, oder Aglaja Veteranyi aus Rumänien - die Gründe der Niederlassung in der Schweiz sind verschieden. Es gibt unter den Einwanderern solche, die hier heiraten, man kommt zum Studieren hierher, und es gibt solche, die als Saisonarbeiter in unser Land gekommen sind wie der Kosovo-Albaner in der Anthologie: Vaxhid Xhellili. Der jiddische Lyriker Lajser Ajchenrand oder der Deutsche Otto Nebel sind als politische Flüchtlinge gekommen.

\section{Und Dragica Rajčić auch?}

Ja. Aber zuerst kam sie nur zum Arbeiten. Sie ist dann wieder zurück nach Kroatien gegangen, und als der Krieg begonnen hat, ist sie definitiv in die Schweiz gekommen. Aber diese Stimmen gehören zur Literatur, zur Poesie in der Schweiz. Ich würde sie aber nicht als Schweizer Autoren im strengen Sinn bezeichnen. Ich meine, wie Sie gesagt haben, es gibt viele Ausländer in der Schweiz, die unsere Gesellschaft und Kultur prägen. Sie bringen einen anderen Ton, eine andere Sichtweise hinein. Aber ich glaube nicht, dass es eine große Beziehung zwischen vielen der eingewanderten Dichter und den Schweizer Autoren gab und gibt.

Rajčić ist übrigens ein interessantes Phänomen. Sie hat ein so genanntes Gastarbeiterdeutsch entwickelt. Das heißt ein Deutsch, das viele Leute aus dem Balkan bei uns sprechen - ein rudimentäres, manchmal fehlerhaftes Sprechen. Sie hat aus diesem eine Kunstsprache gemacht.

\section{Das hat sie uns vorgestellt. Sie hat voriges Jahr in Slowenien Lesungen gehabt.}

Ich habe mit der Anthologie auch eine Veranstaltung mit ihr gemacht, in Basel. Leider schreibt sie keine Lyrik mehr. Ihre Sprache ist kunstvoll. Sie spielt mit den Fehlern. Und da kommt eine neue Qualität hinein. Aber wenn man ihr beim Reden zuhört, ist ihr Deutsch nicht perfekt - ihre Kunstsprache ist also halb gewollt, halb ungewollt.

Sie haben am Anfang schon erwähnt, dass es in Ihrem Kunstkonzept so etwas wie eine Grenzüberschreitung gibt, und dann schreiben Sie auch in ihrem Nachwort, dass man, wenn man über die Moderne spricht, nicht nur Poesie, Literatur, sondern auch Film, die bildende Kunst usw. einbeziehen muss, denn sonst hat man nicht den ganzen Kontext. Es geht sehr viel verloren, wenn man den nicht hat, und da sieht man ja sehr schön, dass das s Konzept diese Grenzüberschreitung thematisiert. 
Das wollte ich schon immer machen. Als ich das Hörbuch Wenn ich Schweiz sage ... edierte, bin ich mit Ingo Starz, dem Mitherausgeber, auf viele Dichter gestoßen. Einige konnten wir nicht aufnehmen, weil es von ihnen keine Aufnahmen gab. Bei den Recherchen habe ich aber auch Autoren entdeckt, die Wort- und Schriftbilder geschaffen haben, die man natürlich in einem Hörbuch nicht präsentieren konnte. Trotzdem war es für mich immer klar, dass diese Bilder wie die eigentliche Lyrik, Prosagedichte oder Songtexte zur Poesie gehören. Dieser Begriff schließt für mich, anders als Lyrik oder Gedicht, auch solche Formen ein. Deshalb steht im Titel auch nicht „Moderne Lyrik“ oder „Moderne Gedichte", weil Poesie für mich einfach ein

\section{umfassenderer Begriff ist?}

Ja, ein umfassenderer Begriff. Obwohl: heute ist eigentlich alles Poesie. Seit ich diesen Begriff gewählt habe, lese ich überall „Poesie“. Das ist ein Begriff, der eigentlich im Übermaß verwendet wird, für alles. Er heißt viel und heißt zugleich nichts. Unter Poesie versteht man ja auch eine bestimmte Qualität zum Beispiel in einem Film oder einem Bild, wobei vom Bezeichneten eine Wirkung ausgeht, die sich der Sprache entzieht. So spricht man von einem ,,poetischen Film“. Ich verwende den Begriff aber im formalen Sinn, als Gefäß auch für grenzüberschreitende Texte. Ich versuchte, diese nicht im Überfluss zu zeigen, sondern nur in ausgewählten Beispielen. Denn wenn man gar keine Begrifflichkeit mehr hat, wird es beliebig. Das ist sicher eine Gefahr. Aber eben: Der Hintergrund meiner editorischen Haltung ist, dass man heute nicht nur von einer Sparte aus denken kann. Viele Sachen sind doch in dem von Ihnen erwähnten Kontext entstanden.

\section{Man kann sie ohne diesen Kontext nicht verstehen?}

Eben. Also, ein Paul Klee hat nicht nur Texte geschrieben, oder Bildtitel, sondern auch Bilder gemacht, welche Dichtung und Sprache thematisieren. Literatur erscheint hier also in bildnerisch-literarischer Form. Das ist das Interessante. Die Germanistik rümpft über solche grenzüberschreitende Werke ein wenig die Nase, immer noch. Doch alle Kritiken der Anthologie waren sehr gut. Einer von der Universität hat kritisiert, dass es vielleicht ins Beliebige laufen kann, wenn man den Begriff so offen hält, dass man alles reinnehmen kann. Das ist nicht ganz falsch, natürlich, aber ich finde, man muss eben auch mutig sein, um Grenzen, auch des Edierens, zu sprengen.

\section{So wie die Künstler die Grenzen sprengen?}

Genau. Um solche Texte zu edieren, konnte es nicht ein Buch sein, das traditionell und risikolos ediert ist und gestaltet wie eine der üblichen Anthologien. 
Ihre Anthologie wurde auch für die Gestaltung ausgezeichnet.

Ja. Das war wichtig. Die Gestaltung beeinflusst die Lektüre stark, sollte sie beeinflussen. Dass man sich nicht überfordert fühlt. Denn wenn man in vielen anderen Anthologien eine Seite nach der anderen blättert, sind sie meistens ohne großes strukturelles Prinzip, gestalterische Finessen und ohne Leerräume von oben bis unten mit Texten gefüllt. Man verliert rasch die Lust zur Fortsetzung der Lektüre und fragt sich, was der Sinn und Mehrwert dieser Anordnung ist. In meiner Anthologie zeigen die Kapitel zum Beispiel auch verbindende Themen, damit der Leser in diesem Ozean von Vieldeutigkeiten und vielen Formen irgendwie einen gemeinsamen Nenner suchen und finden kann. So dass er dadurch auch ein wenig Orientierungs- und Haltepunkte hat.

Wenn man die Gedichte liest, dann spürt man sehr viel Trauer in ihnen. Also ich denke jetzt zum Beispiel an Walser: „Die Unerschütterten, die Sicheren brachten nie etwas Grosses zustande. “ Oder Dürrenmatt: „In unserem Land leben nur die Toten. “ Oder wenn der Schweizer Bob Dylan, Mani Matter, fragt: ,warum seid ihr so traurig?" Und dann gibt es auch einige Autoren, die Selbstmord begingen, und Sie haben vorhin schon erwähnt, viele waren in der Klinik. Robert Walser war in der psychiatrischen Klinik und sehr viele andere, z. B. auch Wölfli.

Drei von vier der als Pioniere bezeichneten Dichter waren in einer psychiatrischen Klinik.

Womit hängt das zusammen, diese Trauer, dass sie in der psychiatrischen Klinik landen und dann so Großes dort schaffen? In der psychiatrischen Klinik sind sie irgendwie von der Außenwelt abgegrenzt?

Es ist eine schwierige Frage. Man muss unterscheiden: Walser ist ein anderer Fall als Wölfli. Wölfli war ein ungebildeter, straffällig gewordener Knecht, der in der Klinik zu schreiben und zu malen begonnen hat. Walser hat lange unauffällig wie die meisten von uns gelebt und ist erst spät in die Klinik gekommen. Das sind zwei verschiedene Fälle. Ich meine einfach, dass die Schweiz in der ersten Jahrhunderthälfte ein sehr kleines, enges, engstirniges Land war. Natürlich die Demokratie, Freiheit ..., aber man musste sich anpassen. Und die, welche nach neuen künstlerischen Formen, auch neuen Lebensformen gesucht haben, sie hatten es schwer in der Schweiz.

Wie z. B. eine Annemarie Schwarzenbach?

Genau. Das Kleine. Man wurde beobachtet, man durfte nicht homosexuell oder lesbisch sein. Da ging man eben nach Berlin, wie Schwarzenbach oder wie Walser. Dort herrschte eine größere Toleranz und man war nahe am Puls der Kultur.

Walser verbrachte bei seinem Bruder, der Maler war, sechs Jahre, die auch seine fruchtbarsten Jahre waren. 
Ja. Das ist auch das Thema von Paul Nizons Diskurs in der Enge, das er heute immer noch verficht. Heute gilt das nicht mehr, aber damals stimmte das schon. Viele wichtige Künstler haben den Durchbruch im Ausland geschafft, weil dort eine fruchtbare Atmosphäre herrschte, es nicht zu viele Vorurteile gab, man konnte sich frei bewegen, frei arbeiten. Und dann kam das Problem der Rückkehr in die Schweiz. Das war bei Walser auch der Fall. Er war zwar in Berlin ein wenig eigenartig oder eigen gewesen, ohne dass er aber deswegen gleich eingesperrt wurde. Die eigentlichen Probleme begannen nach der Rückkehr. Man war an eine offene Lebensführung und an ein tolerantes Umfeld gewöhnt - ein Hans Morgenthaler auch. Dieser war in Thailand gewesen, hatte dort ein freies Leben geführt und kam dann, wie er geschrieben hat, zurück in diese kleine, ,elend enge“ Schweiz, wo man nicht auffallen durfte und mit seinen Büchern wenig verdiente. Wenn man damals ein wenig anders war und gegen gewisse gesellschaftliche Regeln verstieß, landete man manchmal schnell in der Psychiatrie. Natürlich waren diese Menschen auch nicht wie jedermann, sondern sie waren, wie Friedrich Glauser und Annemarie Schwarzenbach, drogensüchtig oder wie Walser ein exzentrischer Einzelgänger. Sie wurden in psychiatrische Kliniken eingewiesen, wo sie, im Gegensatz zu heute, nur unter großen Schwierigkeiten wieder entlassen wurden oder für immer eingesperrt blieben. Andere wie Wölfli und Schwartzlin-Berberat begannen erst in der Klinik, künstlerisch zu arbeiten. Man musste ja etwas machen, um nicht ganz verrückt zu werden. Aber das erklärt noch nicht, weshalb in einem solchen Umfeld derart herausragende Werke entstehen konnten. Denn viele psychiatrische Patienten haben nichts gemacht, konnten wegen ihrer Krankheit gar nichts machen - der Großteil war künstlerisch unbegabt. Aber es ist einfach ein Fakt - man müsste einmal ein Buch machen über das -, eine Tatsache, dass in der Schweiz vor dem Zweiten Weltkrieg es viele Künstler gegeben hat, die in der Klinik waren oder am Rande der Gesellschaft gelebt haben, zu Lebzeiten unbekannt waren und nach ihrem Tod weltbekannt wurden. Auch Maler-Dichter wie ein Louis Soutter oder eine Aloïse, die beide im Buch drin sind.

\section{Sie haben sehr viele doppelt begabte Künstler in Ihre Anthologie aufgenommen.}

Wie vorher gesagt, sie haben eben auch modernere Werke als die Autoren, die sich als Dichter verstanden, geschrieben. Unter diesen grenzüberschreitend arbeitenden Persönlichkeiten findet man viele Art Brut-Künstler. Ich glaube übrigens nicht, dass die vorher geschilderten Schicksale in Großstädten - wie Paris oder Berlin - ebenso ausgeprägt gewesen wären. Das hat schon mit diesem Wechsel - große Welt, Freiheit, Rückkehr in die kleine enge Schweiz - zu tun, was zu großen Spannungen führte, die offenbar bei einigen einen schöpferischen Impuls ausgelöst hat. Diese kleinbürgerliche Atmosphäre in unserem etwas engstirnigen Land herrschte noch lange, bis in die 50er, 60er Jahre. Heute gibt es ein anderes Phänomen - heute ist eigentlich alles möglich und fast nichts mehr anstößig. 
Und wie war es mit Alexander Xaver Gwerder, der mit 29 Jahren Selbstmord verübte? Gwerder ist ein Beispiel für jemand, der sich auch am Militär gerieben hat. Er wollte nicht mehr den obligatorischen Militärdienst leisten. Und er wollte, als Verehrer von Rimbaud, weggehen und sich nicht in die Kleinheit unseres Landes fügen. Er hatte Kontakte zu Deutschland, zu Verlagen und Zeitschriften. Die Auswanderungspläne scheiterten, und er hat es irgendwie doch nicht geschafft, seiner bürgerlichen Existenz zu entkommen. Er hätte es versuchen können. Ich habe seine Frau noch gekannt, und sie hat mir gesagt, sie wäre sogar arbeiten gegangen, damit er eine Schriftstellerexistenz hätte führen können. Aber er hat dann eine junge Frau kennengelernt, in ihr eine Todesgefährtin gesucht, um mit ihr zusammen Selbstmord zu begehen. Das ist misslungen, und er ist in Arles alleine gestorben. Er hat nicht mehr geglaubt, dass es eine Zukunft für ihn gibt. Ja, es gibt diese Art Autoren auch. Zu diesen gehört auch eine Aglaja Veteranyi,

die aus einer Zirkusfamilie stammte, die aus Rumänien geflohen ist und in der ganzen Welt herumreiste. Sie kam ja erst mit 15 in die Schweiz, wo sie richtig lesen und schreiben und Deutsch lernte um dann als Autorin große Anerkennung zu erleben.

Ja. Wobei man nicht genau weiß, weshalb sie Selbstmord verübt hat, weil sie zu diesem Zeitpunkt eine anerkannte Autorin war, auf dem Höhepunkt des Erfolges. Sie war sicher nicht wie Gwerder unglücklich gewesen, weil sie sich eingeengt gefühlt hat, sondern das war ein persönliches Schicksal, eine Tragik, verbunden mit einer besonderen seelischen Konstellation, die zu dieser Tat geführt haben muss. Das ist etwas anderes.

\section{In ihrem Buch Warum das Kind in der Polenta kocht kann man das schon ein wenig spüren.}

Ja. Aber wie Sie sagen, es gibt diese traurigen und verrückten Künstler, es gibt diese Selbstmörder und Außenseiter - und diese spezielle Gattung von Autoren interessiert mich besonders. Ich könnte sie auch weglassen, sagen, ja das ist nur ... Aber ich wollte auch, dass man sieht, dass die Schweiz nicht nur ein Land der Schokoladen und Banken ist.

\section{Wie man im Ausland denkt?}

Ja, welch ein Klischee. Dass die Schweiz eben auch ein Land von zerrissenen Künstlern und Autoren ist, die um etwas gerungen haben. Wo die Trauer oder Trauerarbeit auch eine Rolle spielt. Um dieses Thema abzuschließen: Ich meine, dass diese Art von Künstlern, die psychisch labil waren oder in einer Klinik gelebt haben, zum Beispiel die politischen Spannungen in den dreißiger Jahren - die Nazis, Mussolini usw. - künstlerisch eindrücklicher reflektiert haben als die so genannten „,normalen“ Autoren. Diese haben vielleicht ein flammendes Gedicht zwecks Solidarität mit den Opfern im Spanischen Bürgerkrieg geschrieben, aber in einer literarisch uninteressanten konventionellen Form. 
Ich wollte zeigen, wie eine Annemarie von Matt oder ein Louis Soutter diese Kriegsund Todesangst in ihrer eigenen gefährdeten Existenz gespiegelt sahen und sie überzeugend literarisch verarbeitet haben, so dass in ihren Werken die beklemmende Atmosphäre der Vorkriegszeit unmittelbar auflebt. In den Werken dieser seismographischen Dichter ist die Schweiz nicht verschont geblieben, auch wenn das Land sich später aus dem Krieg heraushalten konnte.

Und die Lyrikerinnen? Wir haben schon über einige gesprochen, aber es gibt ein Kapitel, gewidmet nur Lyrikerinnen. Warum?

Das hat Roman Bucheli von der Neuen Zürcher Zeitung kritisiert. Das sei irgendwie altmodisch. Das sei Feminismus, dass man den Frauen heute noch ein Kapitel widmen muss. Ich verstehe dieses Argument. Ich wollte jedoch einfach ein paar wichtige Schweizer Autorinnen, nicht nur deutschsprachige, sondern auch anderssprachige, gemeinsam in einem Kapitel zeigen, und zwar mit Gedichten zu Themen wie: Liebe, Alter, Tod. Altern und Sterben sind zum Beispiel in den späten Gedichten von Erika Burkart ein wichtiges Thema. Wie geht man poetisch damit um? Ich wollte die literarische Verwandtschaft einiger Lyrikerinnen bezüglich dieser Themen präsentieren. Natürlich findet man Autorinnen auch in anderen Kapiteln. Dort stehen sie aber oft in einem Bezug zu einem Autor und nicht zu einer Autorin. Aber ich möchte diesen Kontext nicht überbewerten.

Ich möchte, dass wir noch zu einer Lyrikerin kommen, zu Leta Semadeni, weil sie auch Gast auf dem Festival Pranger war und wir diese hervorragende Autorin ein wenig kennengelernt haben. Sie haben sie als eine wichtige rätoromanische Autorin aufgenommen.

Ich habe ihre Qualität zuerst nicht gleich erkannt. Ich habe aber auch nicht alles von ihr immer gelesen. Sie ist ein Beispiel für eine eher spätberufene Autorin. Sie ist jetzt 70, und der erste wichtige Band ist vielleicht vor 10, 15 Jahren erschienen. Also war sie dann schon gegen 60. Es ist erstaunlich, dass sie erst seitdem ihre eigene Stimme wirklich gefunden hat. Sie würde das vielleicht anders sehen, aber vorher hat sie gar nicht so viel geschrieben. Nun hat sie ihre eigene Stimme gefunden - mit kargen Bildern, das gefällt mir - es ist kein Wort zu viel, zu wenig. Und dann ist da diese zweisprachige Schreibweise der Gedichte - eine rätoromanische und eine deutsche Version. Wie sie das Entstehen dieser Fassungen erklärt hat, finde ich sehr interessant. Aber ich verstehe ihn immer noch nicht genau, diesen Dichtungsvorgang.

Sie hat erzählt, dass sie das Gedicht einmal auf Deutsch beginnt und einmal auf Rätoromanisch.

Ja, wie man das überhaupt kann. Ich meinte immer, sie hätte zuerst eine Fassung geschrieben und dann vielleicht 


\section{übersetzt?}

Nein, nicht übersetzt eins zu eins, aber vielleicht nochmals in der anderen Sprachversion nach einer ihr gemäßen Form gesucht. Sie aber hat gesagt, sie switche hin und her. Sie geht von einer Fassung, vom Deutschen ins Rätoromanische, vom Rätoromanischen ins Deutsche - und so entsteht ein Gedicht. Das ist schon außergewöhnlich.

Sie hat auch gesagt, dass das Gedicht für sie erst dann fertig ist, wenn beide Fassungen da sind: deutsche und rätoromanische.

Dies lässt sich auch bei einem anderen bedeutenden rätoromanischen Lyriker feststellen, Clo Duri Bezzola. Im Übrigen haben die Rätoromanen wenige wichtige Autoren. Es ist eine kleine Gemeinschaft, und Semadeni ist meiner Meinung nach nicht nur die wichtigste zeitgenössische rätoromanische Lyrikerin, sondern Autorin generell.

\section{Das wurde auch anerkannt, als sie den Schillerpreis bekommen hat.}

Sie hat auch den Bündner Literaturpreis erhalten. Das ist eine wichtige Auszeichnung. Und ich meine, sie hat sie verdient. Ihre Gedichte haben oft einen unerwarteten Schluss. Man denkt sich, was soll das - und der Schluss ist wie ein Paukenschlag. Ihr Schreiben hat manchmal wie eine Bewegung, und dann kommt diese zum Stillstand, oder es gibt daraus eine Folgerung. Und diese Folgerung ist überraschend. Sie hat offenbar schon einige Zeit keine Gedichte mehr geschrieben, jedoch kürzlich ein Romanmanuskript auf Deutsch abgeschlossen. Ich bin gespannt auf diesen Text.

Jetzt noch ein wenig zu technischen Fragen. Wie kann man so ein Riesenprojekt überhaupt durchführen, neben der regulären Arbeit? Wie sind Sie vorgegangen? Oder wie konnten Sie überhaupt diese zwei Tätigkeiten verbinden?

Viel Freizeitarbeit, auch Leidenschaft muss da sein, und es war gut, dass ich einen Termin, eine Deadline hatte, auch von meiner Arbeitgeberin und Mitherausgeberin des Bandes, der Migros, her, denn es hieß: „Das Buch muss im Herbst 2013, vor deiner Pensionierung, raus kommen." Das war gut. Denn wenn man nicht das Messer am Hals hat, dann sagt man: Ja, das kann auch im Frühling sein, oder so... Ich habe übrigens schon als Student an einer Anthologie mitgearbeitet, habe bereits dort über Autoren wie Morgenthaler und Gwerder geforscht. Ich war damals schon vielseitig interessiert, und das Aha-Erlebnis für meine Lyrikbegeisterung war das Museum der modernen Poesie von Enzensberger.

\section{Übrigens: Warum kommt bei Enzensberger in diesem epochalen Werk kein einziger Schweizer vor? \\ Das ist eine gute Frage. Robert Walser oder Blaise Cendrars sind nicht drin, wobei Walser bis in die 60er Jahre nicht bekannt war. Aber Enzensberger hat wirklich den}


Blick geöffnet auf die internationale Poesie, auch editorisch. Der Begriff „Poesie“ ist hier verbürgt. Diese Sammlung war ein wichtiger Auslöser. Ich habe seitdem viel internationale Lyrik gelesen und lese sie immer noch intensiv, also nicht nur deutschsprachige Gedichte, sondern auch russische, amerikanische, spanische, französische usw. Ich liebe aber auch Haiku und klassische chinesische Gedichte. Zu meiner Vorgehensweise: Ich habe Bücher gemacht über verschiedene Autoren wie Morgenthaler, Gwerder, Schwarzenbach, Sonja Sekula oder Nicolas Bouvier. Und wenn man über bestimmte Autoren arbeitet, arbeitet man auch über den Kontext von diesen Autoren. So bin ich auf andere interessante Schriftsteller und Werke gestoßen. Dies alles und die mannigfaltigen Bezüge habe ich irgendwie in meinem Kopf gespeichert.

\section{Und alles vernetzt?}

Ja. Auch alles vernetzt. Ich meine, ohne das Hörbuch hätte dieses Projekt nicht entstehen können, weil ich eben durch die Arbeit daran viele Texte schon im Kopf gehabt habe.

\section{Ich glaube, Sie haben gesagt, dass das, was im Hörbuch ist, nicht in der Anthologie ist?}

Das ist so. Aber ich habe viele Autoren dort schon auf einer Liste gehabt, die ich erst jetzt berücksichtigt habe, weil ich sie im Hörbuch aus irgendwelchen Gründen nicht nehmen konnte. Ich musste nicht bei Null anfangen. Ich wusste auch durch die Arbeit am Hörbuch, wie man so ein großes Projekt realisiert, was man machen muss. Ich habe mit einer Liste gearbeitet, habe alle Autoren aufgeschrieben, die in Frage kommen würden, und habe die Liste immer wieder überarbeitet. Ich habe auch Tipps bekommen, ich kannte natürlich nicht alles. Ein Übersetzer hat zum Beispiel gesagt, du musst Lina Fritschi nehmen, die in Italien lebt, von der ich noch nie gehört habe, und so hat sie Eingang ins Buch gefunden. Man arbeitet nicht allein. Man arbeitet in einem Netzwerk. Aber diese Liste, die ich erstellt habe, das war die Grundlage. Die Liste musste auch schon ungefähr das Verhältnis zwischen deutsch- und fremdsprachigen Teilen enthalten, das heißt, ich wusste etwa, dass die Hälfte deutschsprachig sein kann und der Rest fremdsprachig sein muss. Diese Voraussetzung hat die Auswahl auch bestimmt.

\section{Fremdsprachig heißt hier Italienisch und Französisch?}

Ja. Und Rätoromanisch. Und natürlich dann noch die anderen Sprachen wie die verschiedenen Dialekte, Englisch oder Spanisch. Ich musste auch schon vorher genau wissen, wie viele Texte neu übersetzt werden mussten. Denn um dieses Buch zu machen, musste ich schon vor Beginn der Arbeit mit dem Limmat Verlag zusammen ein Budget erstellen. Im Budget musste ein Posten für das Einholen der Rechte an bestehenden und für die Realisierung von neuen Übersetzungen aufgeführt sein. Dieses Übersetzungsbudget haben wir annähernd eingehalten. Mir war klar, ich durfte nicht einfach machen, was ich wollte, nicht nur meine Liebhaberei ausleben. Ich 
wusste, dass ich nicht alles neu übersetzen konnte. Ich musste gewisse Texte nehmen, die schon übersetzt waren. Trotz der erwähnten Vorarbeiten war das Er- und Zusammenstellen der Kapitel eine Herausforderung, aber irgendwie vorbestimmt. Einzelne Arbeiten haben sich fast magisch angezogen, ich musste nur den ,Vermittler“ spielen. Natürlich habe ich zu viele in Frage kommende Texte von einem Autor gehabt. Wichtig für die Auswahl war: Wie funktionieren sie mit anderen Arbeiten? Gewisse Texte, die mir besser gefallen haben, habe ich weggelassen, weil sie sich nicht so gut eingefügt haben. Ich habe also nicht die schönsten Gedichte, wie eine Anthologie von Peter von Matt heißt (Die schönsten Gedichte der Schweiz), genommen und oft auch nicht die bekannten Texte eines Autors. Diese sind vor allem im Hörbuch enthalten. In erster Linie hat mich interessiert, ob und wie die Texte im Kontext funktionieren, als Zustimmung oder als Widerspruch.

\section{Die Anthologie ist sehr gut aufgenommen worden. Was bedeutet Ihnen das?}

Natürlich freut mich das, wenn ich an den Riesenaufwand für dieses Projekt denke. Der Verlag war sehr gefordert mit diesem für ihn ungewöhnlichen Buch. Mehrere Leute haben ein Jahr lang intensiv an der Realisierung gearbeitet. Neu für mich war: Ich habe nicht ein Manuskript erstellt, wie man es sonst macht, und dann dem Verlag gegeben, sondern das Buch ist Kapitel für Kapitel entstanden. Das heißt, ich habe die ersten fünf Kapitel gemacht, dann diese dem Verlag gebracht, der die Texte gesetzt und die Rechte eingeholt hat. Das Gleiche wiederholte sich mit den folgenden Kapiteln, bis zum Schluss.

\section{Das heißt, Sie hatten von Anfang an diese Anordnung der Kapitel?}

Natürlich hatte ich ein ungefähres Gerüst im Kopf, aber kein genaues. Also z. B. den Schluss wusste ich nicht genau - wie schließe ich ab mit diesen Song-Texten. Ich wusste, dass solche rein müssen, aber ich wusste nicht, welche Songs ich nehmen wollte. Das Konzept, dass ich bekannte Songs von einigen wichtigen Songwritern mit Texten von Spoken-Word-Poeten wie Pedro Lenz verbinde, entstand erst gegen den Schluss.

Wie erwähnt, entstand das Buch schrittweise. In Marathonsitzungen wurden die bereits gesetzten Kapitel, ihre Gestaltung sowie die Auswahl in den folgenden Kapiteln diskutiert. Die am Buch beteiligten Mitarbeiter des Verlages haben übrigens enthusiastisch auf meine Arbeit reagiert: „Das wird ein Jahrhundertbuch.“ Ich habe geantwortet: „Was redet Ihr denn da! Wir können jetzt noch nicht wissen, ob das ein Jahrhundertbuch wird." Obwohl ich wusste, dass ich etwas Neues, Einmaliges machte, war ich eher skeptisch bezüglich Aufnahme und Kritik des Werkes. Ich dachte, dass seine neuartige Struktur, das Weglassen von gewissen bekannten Namen auf Widerstand stoßen würde. 


\section{Wie wurde das Buch auf der Buchmesse in Leipzig aufgenommen, wo die Schweiz dieses Jahr Gastland war und Ihr Buch ein zentrales Ereignis?}

Es war ein Buch unter vielen, aber es war das einzige große nationale Referenzwerk zur Schweiz. Bei der Eröffnung in Leipzig hat der Bürgermeister, das kam im Fernsehen, das Buch in der Hand gehabt. Das war sehr schön. Aber ich habe nicht gedacht, dass es eine solche ausgezeichnete Kritik erhält. Dass fast alle Kritiker und auch meine Herausgeber-Kollegen, die eigentlich Konkurrenten von mir sind, das Besondere der Anthologie erkannt und gerühmt haben, hat mich schon gefreut. Auch in Deutschland gab es positive Reaktionen. Michael Krüger hat eine kleine Laudatio geschrieben im Rahmen der Auszeichnung der Anthologie als einer der schönsten Lyrikbände des letzten Jahres, und Peter Hamm hat das Buch in der Zeit gelobt. Dieser Erfolg macht mich natürlich glücklich, und die gute Aufnahme ist auch ein schönes Abschiedsgeschenk für meine Pensionierung.

Sie sind jetzt in Slowenien, haben auch schon einige Dichter lesen gehört. Sie haben mir gesagt, dass Sie schon im Voraus einiges gelesen haben. Welchen Eindruck haben Sie von der slowenischen Poesie?

Ich kann von denen sprechen, die ich - ich habe zwei, drei Bücher zu Hause von Kosovel, von Šalamun - gelesen habe. Ich habe sie jetzt nochmals vorgenommen, da ich sie einige Zeit nicht mehr gelesen habe, und finde sie zum Teil innovativ, interessant, auch witzig. Dann habe ich auch in die ,lyrikline.org“ rein gehört und habe einige schöne Sachen gefunden. Maruša Krese gefällt mir sehr gut, auch Aleš Šteger kenne ich. Die jungen Autoren, die ich hier auf dem Festival gehört habe, kann ich nur beurteilen, wenn ihre Texte übersetzt wurden - ich finde, diese haben durchaus eine gute Qualität. Da müssen sich die slowenischen jungen Dichter vor den Schweizer Dichtern nicht verstecken. Obwohl ich nicht beurteilen kann, wie repräsentativ diese Dichter sind. Gibt es Dutzende andere, bessere oder gehören sie zu den besten? Und man müsste noch weitere Texte kennen, nicht nur einen pro Autor, das ergibt manchmal ein falsches Bild. Denn es gibt solche, die ein gutes Gedicht geschrieben haben, während die anderen Texte eher mittelmäßig sind. Aber das Niveau fand ich generell sehr gut und vor allem beeindruckt mich das Interesse hier, auch von Seiten der Verlage, dass man überhaupt Poesie veröffentlicht, dass man ein Festival macht für diese Gattung und dass sogar Schüler, Gymnasiasten Gedichte schreiben, die schon recht gut sind. Bei uns findet man derart junge Dichter selten. In unserem Land läuft alles eher über Slam Poetry und Spoken Word. Die jungen Leute machen das oder besuchen solche Veranstaltungen. Nicht unbedingt klassische Dichterlesungen. 